\title{
SISTEMA DE INTERNET DE LAS COSAS (IOT) PARA EL MONITOREO DE CULTIVOS PROTEGIDOS
}

\section{INTERNET OF THINGS (IOT) SYSTEM FOR THE MONITORING OF PROTECTED CROPS}

\author{
Jorge E. Gómez¹, Samir Castaño¹, Teobaldis Mercado², Jose García¹, Alexánder Fernández¹ \\ Recibido para publicación: 4 de febrero 2017 - Aceptado para publicación: 18 de abril 2017
}

\section{RESUMEN}

Este trabajo presenta un sistema de Internet de las cosas para el monitoreo de cultivos protegidos, a través del desarrollo de un sistema con capacidad de recolectar información de parámetros relacionados con el desarrollo y crecimiento de los cultivos. Los datos obtenidos son enviados al servidor para ser procesadas y enviadas al usuario a través de los protocolos y procedimientos del Internet de las cosas (IoT). El propósito es recopilar datos en tiempo real para analizarlos y permitan la toma de decisiones por parte del mismo sistema y el agricultor. El usuario puede interactuar con el sistema de manera remota y recibir las alertas y condiciones especificadas. Los resultados iniciales demuestran que el sistema provee completa información del estado de estos parámetros, ayudando en la tarea del manejo de este tipo de cultivos.

Palabras clave: Cultivos protegidos, IOT, MQTT, agricultura de precisión, control variables.

\begin{abstract}
This work presents an Internet system of things for the monitoring of protected crops, through the development of a system capable of collecting information on parameters related to the development and growth of crops. The obtained data is sent to the server to be processed and sent to the user through the protocols and procedures of Internet of Things (IOT). The purpose is to collect data in real time to analyze them and allow decision making by the same system and the farmer. The user can interact with the system remotely and receive the alerts and conditions specified. The initial results show that the system provides complete information on the state of these parameters, helping in the management of this type of crops.
\end{abstract}

Key words: Protected crops, IOT, MQTT, precision agriculture, variable control.

\footnotetext{
1 Universidad de Córdoba, Departamento de Ingeniería de Sistemas, Montería - Córdoba

2 Universidad de Córdoba, Departamento de Ingeniería de Agronómica, Montería - Córdoba
} 


\section{INTRODUCCIÓN}

Los desarrollos en la ciencia y la tecnología han permitido en los últimos tiempos, mejorar las condiciones de vida de los seres humanos. Esto ha permitido mejorar muchos procesos en la vida diaria de las personas, incluida la agricultura. La agricultura desde los comienzos de la civilización ha forjado al ser humano hasta nuestra época, de hecho la base de la alimentación pasada y actual sigue dependiendo de ella. Dada la importancia de la agricultura desde hace mucho tiempo se viene trabajando en sistemas que permitan optimizar procesos, para mejorar la producción. Estos procesos han estado vinculados con la mejoras genéticas de las plantas, erradicación de plagas, técnicas de siembra entre otros. Los cambios extremos en el clima, en muchas regiones del mundo y en especial las zonas tropicales afectan directamente el comportamiento de la productividad de los cultivos. A raíz de estas situaciones, se han venido desarrollando nuevas tecnologías que deriven en conocer el estado de los cultivos, que van desde las condiciones de humedad del suelo, temperatura, y niveles de radiación. La posibilidad de la incorporación de las tecnologías relacionadas con el Internet de las Cosas (IoT), la recolección de información, el monitoreo y la evaluación de un sistema de cultivo resulta determinante para una efectiva toma de decisiones (Mohanraj I 2012), mejora o corrección de la producción para alcanzar el necesario abastecimiento mundial, aún más, cuando el inadecuado manejo de los recursos hídricos ponen en peligro esta necesidad. (Anaya-Isaza 2016). Los cultivos protegidos dan la posibilidad de controlar el clima y los aspectos físico químicos, para lograr mejorar la productividad (Mohammed Al-Hadithi 2016).

Los avances en las tecnologías de la información y la comunicación (TIC) en la última década, han logrado que estos conceptos importantes, las IoT y Cloud computing se hayan integrado notoriamente en las diversas soluciones en los diferentes campos de acción (Popovic, 2017), como son las Smart Cities, medicina, monitoreo ambiental, seguridad y otros. Debido a esta adaptabilidad y la viabilidad de usar estos avances en el control de los cultivos, es posible a través de las loT integrar objetos físicos con sistemas basados en computadoras (Popovic, 2017), los cuales equipados con sensores de diferentes tipos y adaptados a la tarea Estos sistemas recolectan datos para ser analizados con la finalidad de entender mejor el medio que rodea el cultivo permitiendo visualizar de una forma más clara los aspectos relacionados con el proceso de crecimiento y control de la producción. Lo que se pretende en parte, con este trabajo, es traer a los cultivos protegidos aspectos pertenecientes a los cultivos de precisión, dado que estos buscan el control absoluto de los parámetros que inciden en el desarrollo de las plantas de un cultivo, usando herramientas tecnológicas aplicadas a cultivos extensivos. (Karim 2017) Este articulo busca mostrar como la implementación de un sistema de Internet de las cosas para el monitoreo de cultivos protegidos puede generar un alto nivel de monitoreo, obtenido por el despliegue de un conjunto de dispositivos que recolectan los datos de los parámetros a observar. Para fines de esta investigación los parámetros valorados están relacionados con el porcentaje de humedad del suelo, temperatura ambiental y humedad relativa del aire, así como también el nivel de radiación. El control permanente de estos valores son determinantes en la calidad de los cultivos protegidos, aún más cuando se trate de una variedad foránea de la región, la cual podría tener más complicaciones en su proceso de desarrollo y adaptación. Como se puede deducir en este contexto, un monitoreo y un análisis preciso de los parámetros del cultivo y sus variables de entorno hacen posible asignar de manera adecuada los recursos disponibles para los diferentes tipos de agricultura (Sawant- Durbha 2017), garantizando no solo el control sobre los mismos sino la obtención de las cantidades y calidades requeridas.

La interacción del usuario con el sistema está dada por el manejo de un módulo web, que permite el control permanente y el análisis de los datos, logrando tomar decisiones de forma inmediata. Basado en el flujo de información constante proveniente del sistema, que pueden ser a través de la visualización del comportamiento de los datos recibidos o de las alertas que este mismo genere de forma automática y que podría colocar en riesgo la integridad del cultivo. El resto de este trabajo está organizado de la siguiente forma, por unos trabajos relacionados en el que se muestran los diferentes aportes vinculados con el crecimiento de cultivos, aplicación de técnicas para agricultura de precisión y la incorporación de monitoreo a través del uso de las tecnologías del Internet de las cosas. Otra sección trata de la motivación para este trabajo en la que se muestra 
las iniciativas para el desarrollo del mismo. Luego se muestra la forma como la arquitectura del sistema puede ser aplicada refiriendo los diferentes procesos que se ejecutan para el desarrollo de este sistema. Como resultado se maneja la operación del sistema en alguna de sus fases y la interactividad con los procesos del usuario, así como, finalmente, se muestran las colusiones abordadas para este trabajo.

\section{TRABAJO RELACIONADO}

El uso de nuevas soluciones relacionadas con el IOT entre estos el desarrollo agrícola, ha permitido que con estas herramientas se pueda llegar a planificar y controlar efectivamente los diferentes tipos de cultivos. También, el desarrollo de diferentes técnicas de control y reducción de polución, análisis y estandarización de productos alimenticios, control de la calidad de la tierra y el mejoramiento de la salud pública (Popovic, 2017). El efectivo de control en los cultivos ayudará a mejorar la producción de estos.

Así mismo hoy en las ciudades se puede apreciar el uso de las tecnologías relacionadas con el loT. El rápido crecimiento de algunas urbes hace necesario el desarrollo de una infraestructura de control que ayude a crear caracterizaciones que lleven al mejoramiento de la calidad de vida de sus habitantes.

Como ejemplo de esto están los trabajos encargados implementar plataformas de control ambiental a través del uso de las loT. Aquí tenemos el control de variables medioambientales en áreas urbanas (Gómez et al, 2017), en las que por medio de estaciones de monitoreo se recolecta información y luego es puesta a disposición de la población y las autoridades competentes.

En otras partes del mundo se desarrollan iniciativas que aprovechan este tipo de trabajos y que tienen como propósito el mejoramiento de su capacidad alimentaria.

La aplicación de diversos sistemas como los relacionados con la monitorización para cultivos en invernaderos (Cama-Pinto, 2014), que busca el control de los diferentes parámetros inmersos en el crecimientos de los cultivos a través del uso e implementación de redes de sensores y control de actuadores que ayuden a hacer un efectivo control de estocultivos. Sehace de manerareal el monitoreo de la humedad relativa y temperatura, contenido volumétrico de agua en el suelo y demás factores que inciden el desarrollo de los cultivos protegidos y que podrían ser monitorizados y controlados con sistemas de red de sensores e loT. Este trabajo muestra la implementación y aplicación de una red sensores, el uso, en general de hardware libre y el uso de un modelo de aplicación sensor cloud - server. El usuario obtendría la información, basada en tablas y gráficos relacionados con los parámetros a estudiar. Las conclusiones de este estudio están relacionadas con la facilidad de aplicación y control en los cultivos y presenta los retos obvios de un proceso de producción de bajo costo, frente al alto costo y baja configurabilidad de los sistemas ofrecidos en el mercado. Los resultados se pueden ver en cultivos protegidos en Europa, en especial en el mediterráneo en el que se ha visto un incremento de este tipo de cultivos, después de China (Cama-Pinto. 2014).

En Norte América, podemos ver un ejemplo del uso de sistemas de control de crecimiento a través de imágenes aéreas o satélites, con las que se buscan controlar la altura de los diferentes cultivos en grandes extensiones (Chang y Jung, 2017). Como parte del uso de la agricultura de precisión, aspecto que el presente trabajo referencia para su aplicación en los cultivos protegidos. Este tipo de análisis busca, entre ostros aspectos, encontrar el momento justo para cosechar, siendo esto muy importante, ya que puede ser la diferencia en las cantidades proyectadas.

En el país encontramos estudios enfocados en el desarrollo de aplicaciones destinadas al control y manejo de cultivos usando este tipo de plataformas.

Un ejemplo de implementación en cultivos extensivos, usando elementos de agricultura de precisión es el trabajo relacionado con los sistemas de información enfocados en tecnologías de agricultura de precisión y aplicables a la caña de azúcar (Orozco y Ramírez, 2015). En este se hace una revisión de las tecnologías utilizadas, la gestión de datos y su arquitectura, en este se presenta un sistema que facilita la optimización en las distintas etapas del ciclo de vida de la caña de azúcar. El sistema propuesto en este estudio busca, en consecuencia, la optimización del proceso de la caña de azúcar en Colombia aplicando la agricultura de precisión (AP) en el cultivo de la caña de azúcar. 
Una muestra orientada al cultivo protegido tiene que ver con el desarrollo de un sistema de monitoreo visual para la estimación del balance del agua en cultivos de hortalizas usando cámaras de bajo costo. El uso de este tipo de sensores tiene el objetivo de lograr una estimación precisa del balance de agua, a través de imágenes temporales de los cultivos, pudiendo entregar el porcentaje de cobertura de verdor (PCG). Con esto podemos calibrar la cantidad de agua a irrigar al cultivo, logrando la reducción. El logro de este trabajo consiste en procesar las imágenes temporales a través de un tratamiento algorítmico. Este verifica el crecimiento y la cobertura de verdor de manera constante permitiendo conocer los estados de necesidad de consumo de agua para el cultivo de hortalizas (González-Esquiva, 2017).

En la universidad del Quindio, Colombia, se implementó un sistema de instrumentación para el invernadero de la unidad agro ecológica La Aldana (Muñoz y Buitrago 2011). A través de este sistema se hace la medición de variables físicas como temperatura, humedad relativa y humedad del suelo. Posteriormente estos datos son controlados al interior del invernadero y de manera simultánea se monitorizan inalámbricamente, desde el cual dependiendo de los datos activa de forma autónoma un sistema de control de goteo por histéresis el cual racionaliza el uso del agua del cultivo.

\section{MOTIVACIÓN}

En la actualidad se hace énfasis en la necesidad de encontrar nuevas y mejores maneras para el adecuado manejo de la producción agrícola. Promover nuevos procesos y más eficientes métodos de control para los cultivos, logra mejorar la calidad y el ahorro de los insumos usados en todo el proceso de crecimiento de los diferentes cultivos.

Este trabajo busca desarrollar estos métodos relacionados con el crecimiento de los cultivos, a través del uso de sistemas de control, enfocados en los cultivos protegidos. Existe la necesidad de aprovechar las condiciones dadas por este tipo de cultivos, las cuales permiten el uso de sistemas de control específicos para el monitoreo de sus diferentes variables de desarrollo medioambiental las que inciden en todo el proceso de crecimiento.

Las plantas como seres vivos, son muy sensibles a los factores ambientales como el contenido volumétrico de agua en el suelo, temperatura y humedad relativa así como también la radiación (Cama-pinto, 2017). Esto que permite desarrollar un sistema de Internet de las cosas (IOT), para monitorizar estas variables en los cultivos protegidos. El objeto de esto es recolectar datos que permitan en tiempo real verificar su estado y a la vez pueda generar alertas al usuario que lleve a una adecuada toma de decisiones o acciones automatizadas como suministro de agua al cultivo o algún tipo de control ambiental.

En el proceso y desarrollo de cultivos es necesario implementar estos sistemas con los cuales se pueden integrar tecnologías de las IoT en el contexto de la agricultura. Es posible observar cómo tanto para la agricultura extensiva o en este caso, de invernadero o protegida, el monitoreo y control da como resultado un manejo apropiado. Con todo esto se estaría garantizando resultados favorables que ayudan al mejoramiento mismo de la producción alimenticia tanto en nuestras regiones como en el mundo.

\section{PROPUESTA}

El sistema de Internet de las cosas para el monitoreo de cultivos protegidos se encuentra desarrollado bajo la arquitectura de tipo cliente servidor, la cual se establece en su descripción a través de capas explicadas a continuación.

Capturas de Datos: Esta capa se encarga de obtener los datos de los respectivos sensores, los cuales son recibidos por una estación de control para luego ser llevarlos usando protocolo MQTT a través de un broker que gestiona los topics publicados.

1.1. Administración y Procesos de Capturas: Esta capa es la responsable de la escucha permanente a través de la suscripción de los datos provenientes de los topics publicados para cada sensor. El protocolo MQTT es uno de los protocolos M2M, la cual hace posible la interconexión de los dispositivos en el sistema, esto debido a que permite a sistemas inalámbricos y cableados establecer conexiones. Directamente existe una administración y procesamiento de datos por parte del servidor a través de una interfaz de gestión que recibe la información trasmitida en el sistema. Estos procesos están establecidos como capas subyacentes a los procesos principales. 
1.2. Interfaz de servicios a Clientes: Esta capa se encarga de obtener las peticiones del cliente desde la web móvil, que luego se envía a la capa subyacente es decir a la capa de gestión, para procesar las peticiones procedentes de los clientes. Esta capa también es responsable de mostrar las respuestas a los diferentes clientes mediante HTTP.

1.3. Administración y Gestión: Esta capa está a cargo de la suscripción al broker por un cliente MQTT como Paho, recibe los datos leídos a través del topic de suscrito, y se conecta con la base de datos para su almacenamiento, dependiendo de la variable la cual se esté administrando, ya sea Humedad de suelo, Temperatura, Humedad Relativa y/o Radiación UV. A través de la App se obtienen los datos solicitados por los clientes. El cliente Paho MQTT in- corporado en un script de Python, agregando bibliotecas a la OPS para su implementación. El paquete de datos recibido a través del topic de envío se procesa por medio de cadena, en la que se originan los datos que se almacenan en la base de datos del sistema, y se procede a esperar el siguiente paquete, el intervalo de retardo es determinado por el controlador de los sensores de lectura.

1.4. Recuperación y entrega de información: Esta capa está diseñada para recuperar y entregar información, dependiendo de las solicitudes y respuestas de los usuarios, así como de los sensores. De la misma manera cuenta con algoritmos de información - recuperación para facilitar las consultas realizadas por los clientes. Este despliegue se puede apreciar en la figura 1.

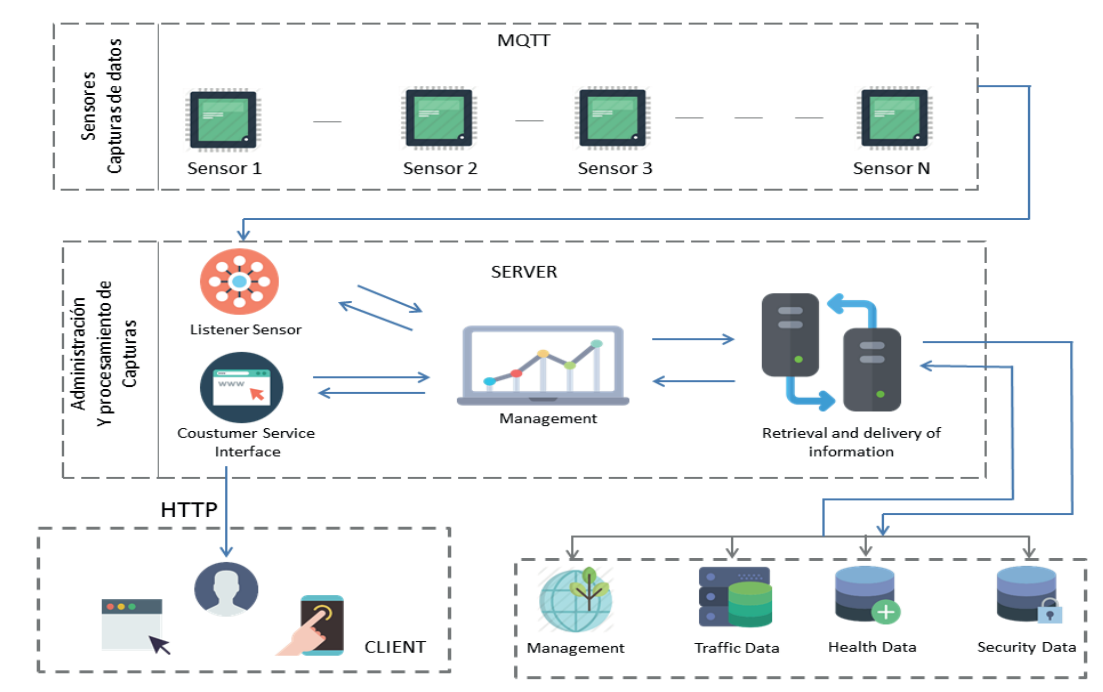

Figura 1. Arquitectura del sistema.

El procesamiento manejado por la estación la cual es la parte del dispositivo que se encarga de la recolección, procesamiento y envío de la información leída a través de los diferentes sensores, y como se muestra en la figura 2, habilita el registro de las variables y el envío de las mismas, procesos que están adaptados a la lectura del sensor correspondiente.

En la figura 3, se encuentra definida la secuencia del proceso de envió de datos o trasmisión, la cual inicia con la lectura de los datos por parte de los

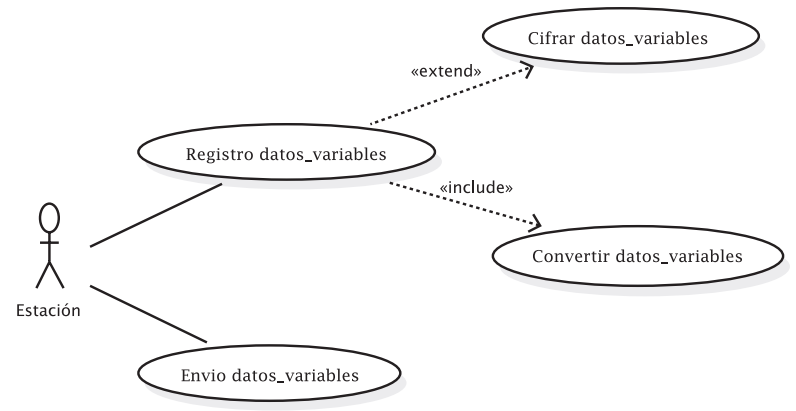

Figura 2. Caso de uso estación 


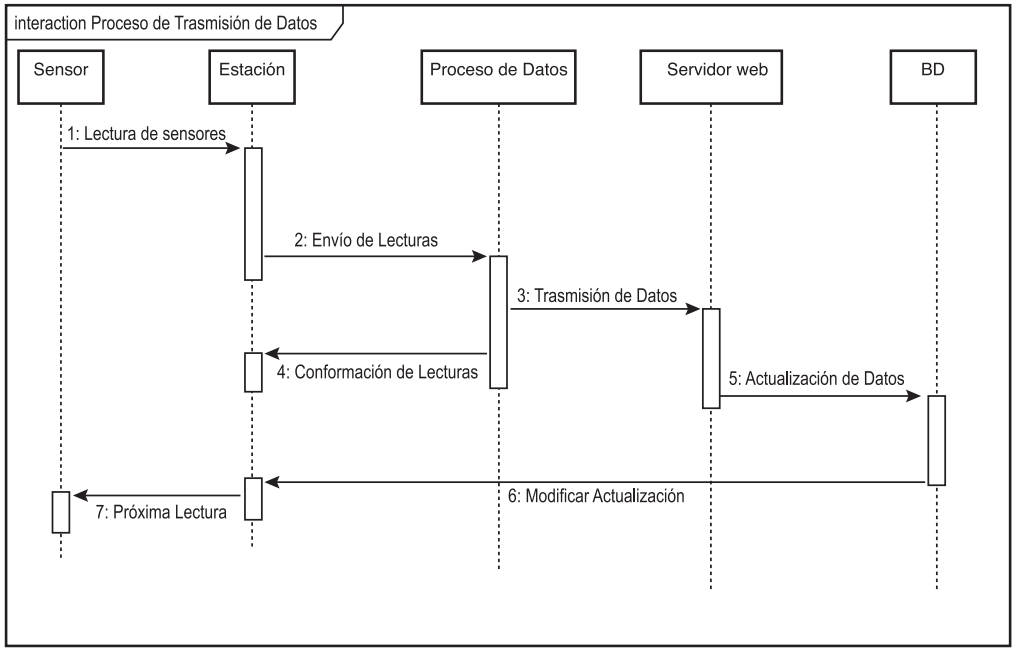

Figura 3. Secuencia para el envío de datos.

sensores, gestión y proceso de datos realizados por la estación. Estos son recobrados para luego gestionar la actualización de la base de datos provenientes de los topics creados para la escucha en el broker matt elegido. La información de la suscripción es incorporada a la base de datos que se actualizada de manera permanente por los sensores, una vez actualizada la base de datos se regresa la respectiva notificación para proceder a la siguiente lectura.

\section{RESULTADOS}

El sistema de Internet de las cosas para el monitoreo de los parámetros obtenidos en tiempo real en un cultivo protegido despliega efectivamente la información obtenida a través del sistema la cual se actualiza automáticamente y es mostrada en la app web que permite al usuario tener los datos ya sea a través del web site o en su móvil. Luego se puede acceder a los diferentes métodos elegidos para visualizar y analizar la información como son tablas con los datos de los parámetros observados o gráficos para el claro analizas de estos.

De igual forma es posible capturar allí las diferentes alertas que tienen como propósito mantener el estado saludable de los cultivos, a través de indicaciones referentes, por ejemplo, a la hidratación o control de temperatura y radiación lumínica, como se pueden apreciar en las figuras 4 y 5 .

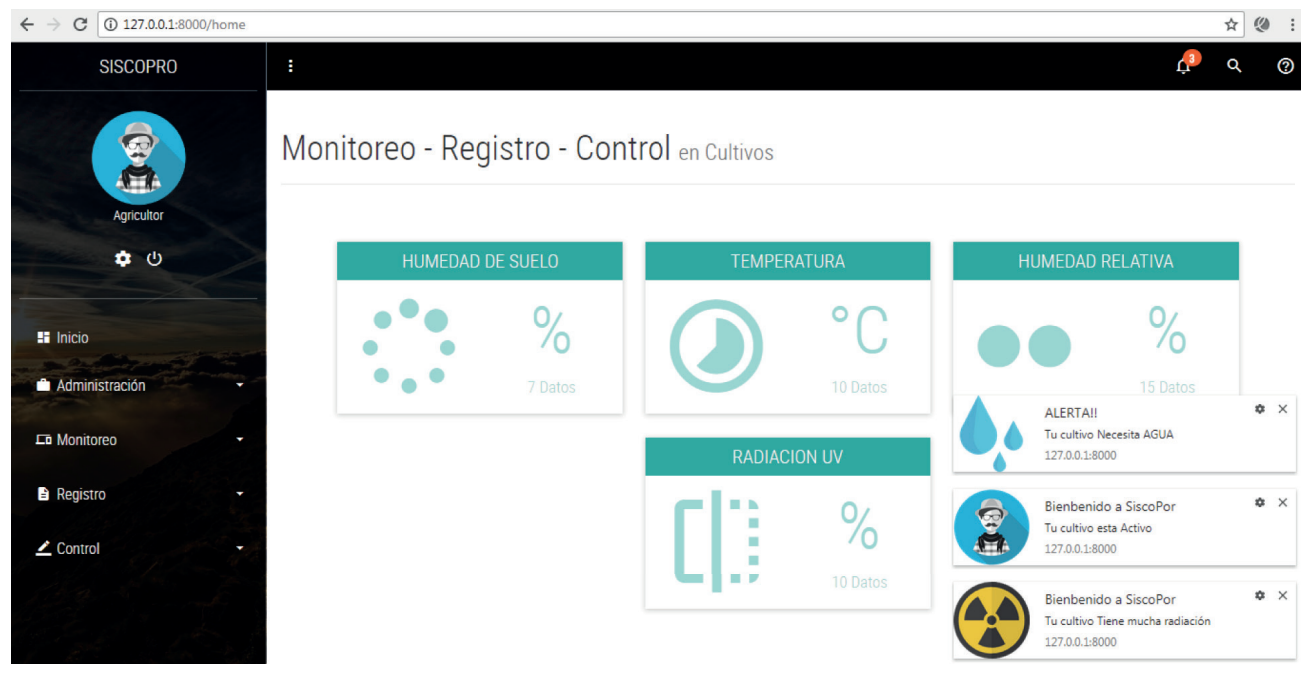

Figura 4. Panel Principal 


\section{Analisis de Variables en Cultivos Protegidos}

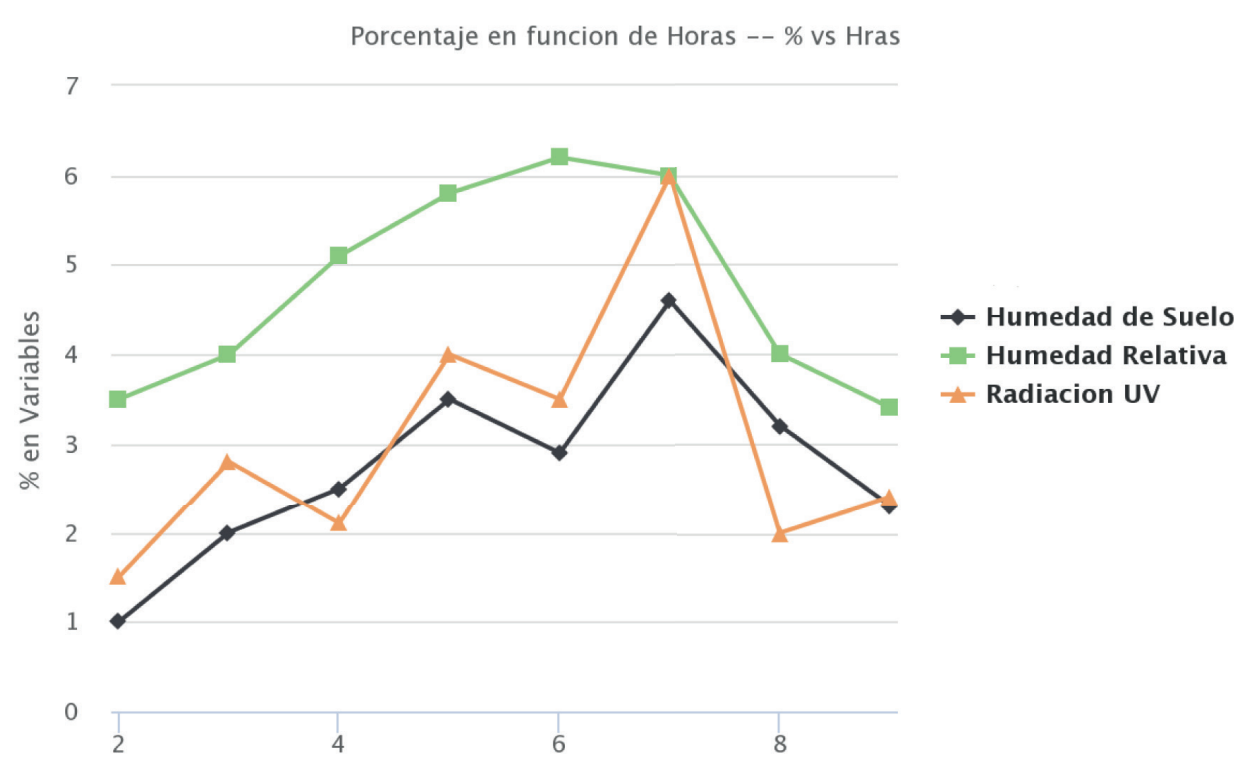

Figura 5. Graficos Parametros

La gráfica porcentaje vs. tiempo muestra el comportamiento de las variables Humedad de suelo, Humedad Relativa y Radiación las cuales permiten analizar observando su variación porcentual en diferentes estados del cultivos con respecto al tiempo transcurrido.

\section{CONCLUSIONES}

En este trabajo se ha propuesto el desarrollo de un sistema de Internet de la cosas para el monitoreo de cultivos protegidos. Los datos fueron efectivamente capturados en tiempo real usando el protocolo MQTT que hace parte de los protocolos M2M, a través de un broker y usando la infraestructura dada por la plataforma de hardware libre Arduino como controlador de los diferentes sensores. Lo que nos permite capturar los datos en tiempo real del porcentaje de humedad del suelo, la temperatura y la humedad relativa del aire, así como también los niveles de radiación que pueden incidir en el desarrollo de los cultivos.

El diseño realizado con este trabajo busca brindar una plataforma que, basado en el IoT, pueda ser usada de manera exitosa en cualquier tipo de cultivo protegido. La información que este sistema obtiene ayuda de manera oportuna a la toma de decisiones que logrará mantener el crecimiento y desarrollo de los cultivos según las metas de producción trazadas.

El bajo consumo de energía y el uso mínimo de la banda ancha del protocolo MQTT hacen que este sistema pueda ser viable ya que permite un bajo coste haciéndolo accesible en especial para las áreas rurales o apartadas.

En trabajos futuros se puede espera la integración de este sistema con plataformas de control automáticos que no solo permitan generar alertas sino que también interactúen de manera automática con el entorno del cultivo protegido. Como ejemplo de esta interacción puede ser la hidratación automática del suelo según necesidad o control ambiental para cultivos foráneos, así como también adaptabilidad de cubiertas para protección de la radiación solar.

\section{AGRADECIMIENTOS}

Esta de investigación fue financiada con recursos de la Universidad de Córdoba y asignados al grupo de investigación SOCRATES, mediante el proyecto -FI-06-16. De igual forma se agradece al programa de Ingeniería Agronómica por su apoyo constante a este proyecto. 


\section{REFERENCIAS}

[1]. Cama-Pinto, A., \& Gil-Montoya, F., \& Gómez-López, J., \& García-Cruz, A., \& Manzano-Agugliaro, F. (2014). Sistema inalámbrico de monitorización para cultivos en invernadero. Dyna, 81 (184), 164-170.

[2]. Anaya-Isaza, A., 2016., Sistema de Riego Basado En La Internet De Las Cosas (IoT). Pag 1.

[3]. Chang, A., Jung, J., Maeda, M. M., Landivar, J., 2017. Crop height monitoring with digital imagery from Unmanned Aerial System (UAS). Computers and Electronics in Agriculture (141) 232-237.

[4]. Mohammed, B., Al-Hadithi, García Cena,C., Cedazo León,R., Loor. C.,2016. Desarrollo de un Sistema de Iluminación Artificial Inteligente para Cultivos Protegidos. p. 421.

[5]. Üstündag.B., (2017). An adaptative machine model for monitorin crop status. 16(2), 252-265.

[6]. Karim,F., Karimb,F., Frihida, A. 2017. Monitoring system using web of things in precision agriculture. The 12th International Conference on Future Networks and Communications. (110) p. 402-409.

[7]. González-Esquiva, J.M., Oates, M.J. G. García-Mateos, B. Moros-Valle, J.M. Molina-Martínez, A. Ruiz-Canales. 2017. Development of a visual monitoring system for water balance estimation of horticultural crops using low cost cameras. Computers and Electronics in Agriculture 141 (2017). p. 15-26.

[8]. Gómez,J., Marcillo,F., Triana,F., Gallo, V., Oviedo, B., Hernández.V. 2017. IOT FOR ENVIRONMENTAL VARIABLES IN URBAN AREAS. The 8th International Conference on Ambient Systems, Networks and Technologies. Procedia Computer Science 109C(2017) 67-64.
[9]. Mohanraj, I \& Ashokumar, Kirthika \& Naren, J. (2016). Field Monitoring and Automation Using IOT in Agriculture Domain. Procedia Computer Science. 93. 931-939. 10.1016/j. procs.2016.07.275. Li Tan. 2016. Cloudbased Decision Support and Automation for Precision Agricultuire in Orchards. IFACPapersOnLIne 49-16(2016) 330-335.

[10]. Orozco, Óscar Arley, \& Llano Ramírez, Gonzalo. (2016). Sistemas de información enfocados en tecnologías de agricultura de precisión y aplicables a la caña de azúcar, una revisión. Revista Ingenierías Universidad de Medellín, 15(28), 103-124. https://dx.doi.org/10.22395/rium.v15n28a6

[11]. Muñoz, P., \& Buitrago, J., \& Arboleda, A., \& Cortes, O., \& Sánchez, A., \& Zapata, C. (2011). Sistema de instrumentación y monitoreo para el invernadero la Aldana de la universidad del Quindío.. Scientia Et Technica, XVI (49), 219-225.

[12]. Suryakant Sawant, Surya S. Durbha, Adinarayana Jagarlapudi, Interoperable agro-meteorological observation and analysis platform for precision agriculture: A case study in citrus crop water requirement estimation, In Computers and Electronics in Agriculture, Volume 138, 2017, Pages 175-187, ISSN 0168-1699, https://doi. org/10.1016/j.compag.2017.04.019.

[13]. Oksanen, Timo \& Linkolehto, Raimo \& Seilonen, llkka. (2016). Adapting an industrial automation protocol to remote monitoring of mobile agricultural machinery: a combine harvester with IOT. IFAC-PapersOnLine. 49. 127-131. 10.1016/j.ifacol.2016.10.024.

[14]. Tomo, P. \& Nedeljko, L. \& Pesic, Ana \& Zecevic, Z. \& Krstajic, B. \& Đukanović, Slobodan. (2017). Architecting an loTenabled platform for precision agriculture and ecological monitoring: $A$ case study. Computers and Electronics in Agriculture. 2017. 255-265. 10.1016/j. compag.2017.06.008 